\title{
Microwave-assisted Extraction for the Chromatographic Analysis of Propellant
}

\author{
Xiaoyu Zhou, Ran Xiong a , Bin Zhang, Yan Luo \\ The 63981 unit of PLA. China \\ a Bxiongran@163.com
}

Keywords: Propellant, UAE, SDWP, Stabilizer, Gas chromatography.

\begin{abstract}
In order to timely and accurate understanding of propellant in the process of storage, security, need for stabilizer determination of propellant, the microwave-assisted extraction (UAE) and solvent-dissolve-water-precipitation extraction (SDWP) were used in this paper for chromatographic analysis of propellant, comparing the result of the test, the error is small, get the microwave-assisted extraction can be used in the gas phase chromatography determination of propellant stabilizer experiment, this method shortens the test time, improve the test efficiency.
\end{abstract}

\section{Introduction}

Propellant is a major energy source for launching projectiles or propelling the rocket movement. It is mainly stored in the warehouse, and its storage security is directly related to wartime use. In the process of storage, the nitrifying cellulose will automatically decompose, which can delay or inhibit the reaction of the reaction when adding the tranquilizer, thus improving the chemical stability of propellant. As a result, the stabilizer can display prolong the storage life of propellant, regular inspection of stabilizer in the propellant, can grasp the quality situation of propellant, so as to guide the management of ammunition properly, use and storage. The microwave-assisted extraction (UAE) and solvent-dissolve-water-precipitation extraction(SDWP) were used in this paper from many traditional powder sample pretreatment method, compare the result of the microwave-assisted extraction to the feasibility of the application of chromatographic analysis for propellant.

\section{Test method}

\subsection{Sample preparation}

A single base propellant: dense texture, with a glass slide; A double - based propellant: dense texture, with a slide of glass; A three - base propellant: dense texture, with slides blown into a flower; Some propellant: the texture is more dense, use glass to scrape the flake. Powder sample pieces: the principle of combustion layer thickness is not more than $0.5 \mathrm{~mm}$ medicine take whole grain, grain burning layer thickness is greater than $0.5 \mathrm{~mm}$ medicine grain processing into small piece of $2-3 \mathrm{~mm}$, $3 \mathrm{~mm}$ and $2 \mathrm{~mm}$ double screen, take on the $2 \mathrm{~mm}$ sieve sieve.

\subsection{Instruments and Reagents}

Reagent: propanone: analysis of pure GB686-78; Petroleum ether: pure HG3-1006-76, boiling point $60{ }^{\circ} \mathrm{C} \sim 90{ }^{\circ} \mathrm{C}$;

Instrument: gas chromatograph: sp-2304 gas chromatograph, as shown in Fig.1; Ultrasonic cleaning instrument: $\mathrm{KH}-3200 \mathrm{~B}$ type ultrasonic cleaner, as shown in Fig.2. 


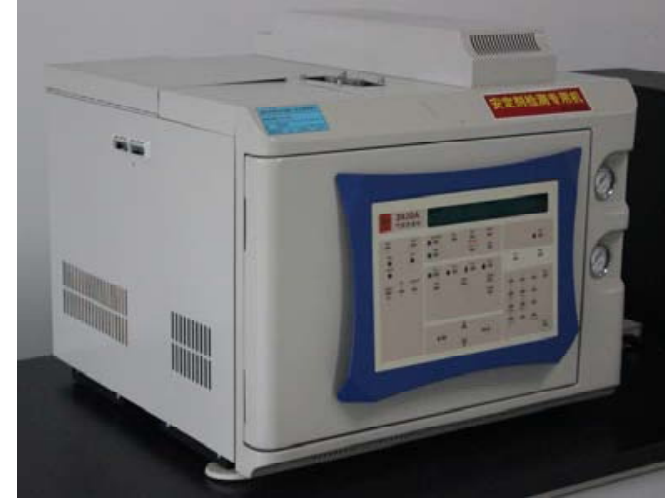

Fig.1 Gas chromatograph 3420A

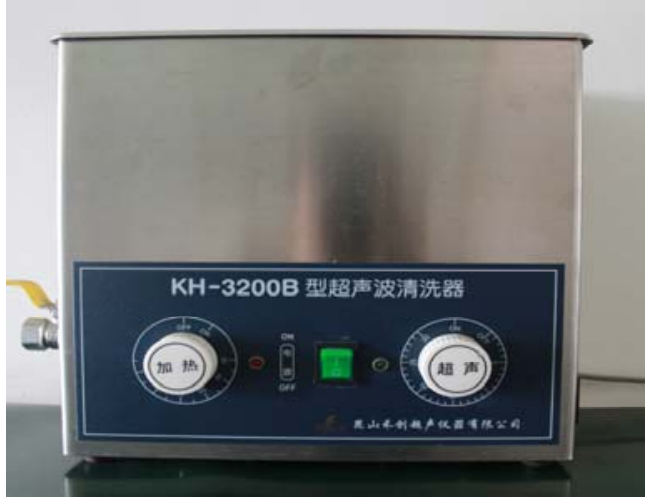

Fig.2 Ultrasonic cleaner

\subsection{The chromatographic conditions}

Carrier gas: high purity hydrogen, flow rate $200 \mathrm{~mL} / \mathrm{min} \sim 240 \mathrm{~mL} / \mathrm{min}$;

Column temperature: $155{ }^{\circ} \mathrm{C} \sim 160{ }^{\circ} \mathrm{C}$;

Gasification temperature: $250{ }^{\circ} \mathrm{C}$.

Bridge current: $180 \mathrm{~mA} \sim 200 \mathrm{~mA}$;

Chromatographic separation column: $3 \mathrm{~mm}$ diameter, $500 \mathrm{~mm}$ stainless steel column.

\subsection{Soak}

Solvent-dissolve-water-precipitation extraction: Said to take the crushed sample $1.5 \mathrm{~g} \sim 2 \mathrm{~g}$ (called a quasi to $0.001 \mathrm{~g}$ ), in the $50 \mathrm{ml}$ of the clean with triangle in a bottle, quantitative add fluid tube with a pipette or add $15 \mathrm{ml}$ volume for (single-base medicine for 4:6, double-base medicine for 3:7) acetone - petroleum ether mixture, after soaking $2.5 \mathrm{~h} \sim 4 \mathrm{~h}$, can be determined.

Microwave-assisted extraction: Said in the crushed sample $1.5 \mathrm{~g} 2 \mathrm{~g}$ (called a quasi to $0.001 \mathrm{~g}$ ), in the $50 \mathrm{ml}$ of the clean with triangle in a bottle, add about $80 \mathrm{ml}$ of acetone ultrasound $20 \mathrm{~min}$ (power density is about $0.2 \mathrm{w} . \mathrm{cm}-2$ ), after transfer into $100 \mathrm{ml}$ volumetric flask and constant volume of water, the filtrate is the sample solution (about $0.5 \mathrm{~h}$ ) sample preparation time, can be determined.

\subsection{Standard solution preparation}

The standard solution is prepared by 1.4 .

\subsection{Calibration}

The instrument was normal and the baseline was flat, the standard solution of $6 \mu \mathrm{L}$ was taken with a microsyringe and the chromatograph was injected to measure the peak height of the stabilizer. The peak height difference of 3 times is not greater than $5 \%$.

\subsection{Determination}

After the calibration, the sample was injected with a microsyringe $4 \mu \mathrm{L}$, injected with the chromatograph, and measured the peak height of the tranquilizer. The difference of the peak height was not greater than 5\%. After testing $2 \sim 3$ samples, the calibration is done

\section{Results calculation and analysis}

\subsection{Calculation formula}

Using formula (1) to calculate the content of the tranquilizer:

$$
C_{i}=\frac{W_{0} \times H_{i}}{W_{i} \times H_{0}} \times C_{0} \times 100 \%
$$

Type:

$\mathrm{Ci}$ - - The content of the tested sample, \%;

$\mathrm{C} 0$ - - The content of the standard propellant, $\%$

$\mathrm{Hi}-$ - The peak of the tested sample;

$\mathrm{H} 0$ - - The peak of the standard propellant;

$\mathrm{Wi}$ - - The quality of the tested samples, g;

W0 - - The quality of the standard propellant, g; 


\subsection{Error provision}

Each sample was prepared with two bottles of leaching fluid, the result was accurate to $0.01 \%$, the difference between the two measurements was not more than $0.1 \%$, and the sample results were averaged.

\subsection{Test results and analysis}

The single-base, double-base, sanki-base and propellant were used to gas chromatography determination of propellant stabilizer test, and the formula (1) is used for calculation, test results and calculation results are shown in table 1

Table 1. the test data of propellant tranquilizers for gas chromatography

\begin{tabular}{cccc}
\hline Sample & Stabilizer content (\%) (UAE) & Stabilizer content (\%) (SDWP) & Error \\
\hline Single-base & 1.50 & 1.58 & $-5.1 \%$ \\
Double-base & 2.65 & 2.54 & $4.3 \%$ \\
Sanki-base & 1.40 & 1.33 & $5.3 \%$ \\
Propellant & 3.15 & 3.08 & $2.3 \%$ \\
\hline
\end{tabular}

Through the content of the stabilizer in the powder in the table 1 shows that the UAE and the SWDP to get the error were within $6 \%$, the error is small, thus, the UAE can be used for the powder of chromatographic analysis.

\section{Conclusion}

1)The UAE was uesd to chromatographic analysis of propellant, the test data compared with the SWDP, the error is small, thus, the UAE can be used for chromatographic analysis of propellant.

2)The extraction time of the UAE was $0.5 \mathrm{~h}$, the SWDP requires 3 hours, so the UAE can shorten the extraction time of propellant, so as to improve the efficiency of the analysis of propellant.

\section{References}

[1] General equipment department general equipment protection department. Gunpowder test [M]. Beijing: National defense industry press, 2000.

[2] General equipment department general equipment protection department. General theory of ammo detection [M]. Beijing: National defense industry press, 2000.

[3] Li dongyang et al. Analysis design and test of ammunition storage reliability [M]. Beijing: National defense industry press, 2013.

[4] Sample pretreatment techniques for chromatographic analysis of energic materials [J]. Energic materials, 23 (3): 279-284. 